UPR-939-T

hep-ph/0105010

\title{
From a Thick to a Thin Supergravity Domain Wall
}

\author{
Francisco A. Brito, Mirjam Cvetič and SangChul Yoon \\ Department of Physics and Astronomy, \\ University of Pennsylvania, Philadelphia PA 19104-6396, USA
}

November 13, 2018

\begin{abstract}
Within $\mathrm{D}=4 \mathrm{~N}=1$ supergravity theory we obtain an effective theory of the thin wall limit for a flat domain wall configuration, interpolating between isolated supersymmetric extrema of the matter potential. We focus on the $Z_{2}$ symmetric flat wall and derive the supersymmetric effective action both in the bulk and on the wall; in the thin wall limit the scalar field, forming the wall, is frozen, and provides the delta function domain wall source, while its fermionic partner decouples due to its large mass. In addition, the interaction between the gravitational supermultiplet and the interaction of the matter multiplet decouples on the wall. While the results are explicitly demonstrated within $N=1 D=4$ supergravity, we also provide a generalization of the result to $D$-dimensions.
\end{abstract}




\section{Introduction}

Domain wall configurations are of special interest in gravity theory, since due to its minimal co-dimension, they are expected to exhibit strong and nontrivial gravitational effects. (See e.g., [1] for a review and references therein.) In particular, flat domain wall configurations are of special interest, since (within $D=4 N=1$ supergravity) they were shown [2] to correspond to supersymmetric (BPS) configurations, interpolating between supersymmetric isolated extrema of the matter potential.

In particular the thin wall limit of the $Z_{2}$ symmetric flat domain wall configuration, interpolating between negative cosmological constant isolated extrema in $D=5$ dimensions, has been a focus of intense study since it allows for trapping of gravity [3] on the wall. In the original version the fixed relationship between the energy density of the wall and the cosmological constant of the bulk was viewed as a fine-tuning condition. It was further pointed out that this condition is related to the BPS mass-density formula for a $Z_{2}$ symmetry BPS limit [2, 4, 5].

The purpose of this paper is to explore in detail the thin wall limit of a flat $Z_{2}$ symmetric domain wall within $D=4 N=1$ supergravity theory. In particular, we will determine the effective action for such a flat wall configuration as one approaches the thin wall limit. We shall identify the bulk and the domain wall part of the action both for the bosonic and fermionic sector of the theory and discuss the properties of supersymmetry transformations in this limit. Details are given in Section 2.

In the second part we shall generalize the procedure to an "effective" supergravity theory in D-dimensions, following Ref.[6]: in the gravity supermultiplet the turned on fields are chosen to be the graviton and gravitino, and in the matter supermultiplet the scalar field (forming a classical kink solution) and the spin-one half partner field are turned on. The details of deriving the thin wall limit effective Lagrangian are given in Section 3. Conclusions are given in Section 4.

\section{BPS Thin Wall Limit Effective Lagrangian within $\mathrm{N}=1 \mathrm{D}=4$ Supergravity}

The starting point is $N=1 D=4$ Lagrangian with one neutral chiral supermultiplet whose superpotential allows for a formation of the $Z_{2}$ symmetric kink solution, forming a BPS domain wall, first discussed in [2]. We first recapitulate these results. Then we turn to the derivation of the thin domain wall and further derive the effective supersymmetric action associated with this thin wall BPS domain wall configuration. 
The $N=1 D=4$ supergravity Lagrangian with one neutral chiral supermultiplet is of the form $([\overline{7]})$ :

$$
\begin{aligned}
e^{-1} \mathcal{L}= & -\frac{1}{2} R-\left|\partial_{z} \phi\right|^{2}-i \bar{\chi} \bar{\sigma}^{m} \mathcal{D}_{m} \chi+\epsilon^{k l m n} \bar{\psi}_{k} \bar{\sigma}_{l} \tilde{\mathcal{D}}_{m} \psi_{n} \\
& -\frac{\sqrt{2}}{2} \partial_{z} \bar{\phi} \chi \sigma^{m} \bar{\sigma}^{z} \psi_{m}-\frac{\sqrt{2}}{2} \partial_{z} \phi \bar{\chi} \bar{\sigma}^{m} \sigma^{z} \bar{\psi}_{m} \\
& +\frac{1}{4}\left[i \epsilon^{k l m n} \psi_{k} \sigma_{l} \bar{\psi}_{m}+\psi_{m} \sigma^{n} \bar{\psi}^{m}\right] \chi \sigma_{n} \bar{\chi}-\frac{1}{8} \chi \chi \bar{\chi} \bar{\chi} \\
& -e^{\frac{K}{2}}\left\{\bar{W} \psi_{a} \sigma^{a b} \psi_{b}+W \bar{\psi}_{a} \bar{\sigma}^{a b} \bar{\psi}_{b}+i \frac{\sqrt{2}}{2} D_{\phi} W \chi \sigma^{a} \bar{\psi}_{a}+i \frac{\sqrt{2}}{2} D_{\bar{\phi}} \bar{W} \bar{\chi} \bar{\sigma}^{a} \psi_{a}\right. \\
& \left.+\frac{1}{2} \mathcal{D}_{\phi} D_{\phi} W \chi \chi+\frac{1}{2} \mathcal{D}_{\bar{\phi}} D_{\bar{\phi}} \bar{W} \bar{\chi} \bar{\chi}\right\} \\
& -e^{K}\left\{\left|D_{\phi} W\right|^{2}-3|W|^{2}\right\} .
\end{aligned}
$$

Here $\left(e_{m}^{a}, \psi_{m}\right)$ denotes the supergravity multiplet components and $(\phi, \chi)$ the chiral superfield components. We have set $M_{p l}=1$. (For notation and conventions see [7].)

The covariant derivatives are defined in the standard form:

$$
\begin{aligned}
& \mathcal{D}_{m} \chi=\partial_{m} \chi+\chi \omega_{m}-\frac{1}{4}\left(K_{\phi} \partial_{m} \phi-K_{\bar{\phi}} \partial_{m} \bar{\phi}\right) \chi \\
& \tilde{\mathcal{D}}_{m} \psi_{n}=\partial_{m} \psi_{n}+\psi_{n} \omega_{m}+\frac{1}{4}\left(K_{\phi} \partial_{m} \phi-K_{\bar{\phi}} \partial_{m} \bar{\phi}\right) \psi_{n} \\
& D_{\phi} W=W_{\phi}+K_{\phi} W, \quad W_{\phi}=\frac{\partial W}{\partial \phi}, \quad K_{\phi}=\frac{\partial K}{\partial \phi} \\
& \mathcal{D}_{\phi} D_{\phi} W=W_{\phi \phi}+K_{\phi \phi} W+2 K_{\phi} D_{\phi} W-K_{\phi} K_{\phi} W
\end{aligned}
$$

The specific form of the superpotential $W$ and the minimal Kähler potential $K$ is chosen to be of the form:

$$
W=\lambda\left(\frac{\phi^{3}}{3}-\eta^{2} \phi\right), \quad K=\phi \bar{\phi} .
$$

This choice allows for a BPS domain wall configuration Ansatz interpolating between two isolated supersymmetric extrema of the potential determined by the solution: $D_{\bar{\phi}} \bar{W}=0$ :

$$
\begin{aligned}
D_{\bar{\phi}} \bar{W} & =\lambda\left[\frac{1}{3}|\phi|^{2}\left(\bar{\phi}^{2}-3 \eta^{2}\right)+\bar{\phi}^{2}-\eta^{2}\right]=0 \\
\phi_{ \pm} & = \pm\left[\frac{3 \eta^{2}-3+\sqrt{9 \eta^{4}-6 \eta^{2}+9}}{2}\right]^{\frac{1}{2}} .
\end{aligned}
$$

The domain wall Ansatz for the scalar component of the chiral supermultiplet and the metric are respectively $\operatorname{Re} \phi=\varphi(z)$ and:

$$
d s^{2}=e^{2 A(z)} \eta_{\mu \nu} d x^{\mu} d x^{\nu}+d z^{2}, \quad \mu, \nu=0,1,2
$$


We employ the Killing spinor equations: $\delta \chi=0$ and $\delta \psi_{m}=0$. Here the gravitino transformation takes the form:

$$
\delta \psi_{m}=2 \mathcal{D}_{m} \epsilon+i e^{\frac{K}{2}} W \sigma_{m} \bar{\epsilon}
$$

and the transformation on the fermionic partner is of the form:

$$
\delta \chi=i \sqrt{2} \sigma^{m} \bar{\epsilon} \partial_{m} \phi-\sqrt{2} e^{K / 2} K^{\phi \bar{\phi}} D_{\bar{\phi}} \bar{W} \epsilon
$$

For BPS supergravity domain walls, first obtained in [2], the equation for $\varphi(z)$ and $A(z)$ arise as solutions of the Killing spinor equations which take the following form:

$$
\partial_{z} \phi(z)=i e^{i \theta} e^{\frac{K}{2}} K^{\phi \bar{\phi}} D_{\bar{\phi}} \bar{W}
$$

with the two component spinor $\epsilon=\left(\begin{array}{l}\epsilon_{1} \\ \epsilon_{2}\end{array}\right)$ satisfying $\bar{\sigma}^{z} \bar{\epsilon}=e^{-i \theta} \epsilon$, where the phase $\theta$ satisfies the following "geodesic" equation:

$$
\partial_{z} \theta=-\operatorname{Im}\left(K_{\phi} \partial_{z} \phi\right) \simeq 0
$$

and is satisfied for the Ansatz $\operatorname{Re} \phi(z)=\varphi(z), \theta=\frac{\pi}{2}$. The warp factor satisfies the following equation (arising from: $\delta \psi_{t}=0$ ):

$$
\partial_{z} A(z)=e^{A(z)} e^{\frac{K}{2}} W
$$

Note again, that while $\phi$ is a complex field, only its real component $\operatorname{Re} \phi \equiv \varphi(z)$ is turned on as a kink solution. This Ansatz is indeed compatible with the Killing spinor equations, thus preserving $\frac{1}{2}$ of the original supersymmetry. (See also [2] for further details.)

The solution was solved numerically in [2] and has the following properties: the scalar field $\varphi(z)$ is a typical kink approaching exponentially fast the asymptotic values $\varphi(z \rightarrow \pm \infty)=\phi_{ \pm}$, given by $(\mathbb{4})$. The warp factor of the metric approaches exponentially fast the Cauchy horizon of the anti-deSitter (AdS) space times of the supersymmetric ground states on either side of the wall, i.e. $e^{2 A(z)} \sim 4 / \sigma^{2} z^{2}$ as $z \rightarrow \pm \infty$, where $\sigma=(4 / 3) \lambda \eta^{3}$.

We now turn to the thin wall limit by taking $\lambda \rightarrow \infty, \eta \rightarrow 0$, and $\lambda \eta^{3} \rightarrow$ fixed. We shall first focus on the bosonic part of the effective Lagrangian in the thin wall background. In this limit, eq.(田) implies that $\phi_{ \pm} \simeq \pm \eta$. In this case the kink solution of (8) assumes the form:

$$
\varphi(z) \simeq \eta \tanh \lambda \eta z
$$


i.e. it approaches the step function $\varphi(z) \simeq \eta \operatorname{sign} z$, whose width goes to zero. We introduce some useful "identities":

$$
\begin{aligned}
& W \simeq-\frac{2}{3} \lambda \eta^{3} \operatorname{sign} z, \quad D_{\phi} W \simeq-2 \eta \delta(z), \quad \partial_{z} \phi \simeq 2 \eta \delta(z) \\
& {\left[\partial_{z} \phi(z)\right]^{2}=\left[D_{\phi} W\right]^{2} \simeq \sigma \delta(z), \quad \mathcal{D}_{\phi} D_{\phi} W \simeq 2 \lambda \eta \operatorname{sign} z, \quad|W|^{2} \simeq \frac{4 \lambda^{2} \eta^{6}}{9} .}
\end{aligned}
$$

(Of course the expressions with the delta functions make sense only under integration.) We can now see easily that the solution (11) satisfies the thin wall limit of the equation (8). It is also instructive to see that in this limit the equation (10) for the metric becomes simply

$$
\partial_{z}\left[e^{-A(z)}\right] \simeq-W \simeq \frac{2}{3} \lambda \eta^{3} \operatorname{sign} z=\frac{1}{2} \sigma \operatorname{sign} z
$$

which has the "exact" solution $e^{2 A(z)} \simeq 4 / \sigma^{2} z^{2}$. Note that the warp factor here agree with the asymptotic behavior discussed earlier.

In the thin wall limit the supersymmetric transformation (6) has the factor of the second term in the transformation, $e^{\frac{K}{2}} W \sigma_{m}$, approaching the step function with the finite width:

$$
\delta \psi_{m} \simeq 2 \mathcal{D}_{m} \epsilon-i\left(\frac{1}{2} \sigma \operatorname{sign} z\right) \sigma_{m} \bar{\epsilon}
$$

Note that since $W$ changes the sign, i.e. it is approximately a step function with a finite width, the effective gravitino mass term in the Lagrangian takes the form:

$$
\mathcal{L}_{\psi^{2}}=\frac{1}{2} \sigma \operatorname{sign} z\left(\psi_{a} \sigma^{a b} \psi_{b}+\bar{\psi}_{a} \bar{\sigma}^{a b} \bar{\psi}_{b}\right)
$$

i.e. on the either side of the wall it has an opposite sign. Note that this result is in agreement with the modified supergravity transformation employed by [8].

Similarly, transformation (7) takes the form:

$$
\delta \chi \simeq 2 \sqrt{2} \eta\left(i \sigma^{z} \bar{\epsilon}+\epsilon\right) \delta(z)
$$

i.e. there is no contribution from the bulk and on the wall the prefactor goes to zero.

We shall now write down the structure of the thin wall effective action. For the bosonic sector, using the results (12), the action becomes:

$$
S^{e f f}=\int_{b u l k} d^{4} x e\left[-\frac{R}{2}+\Lambda\right]+T \int_{b r a n e} d^{4} x e \delta(z) .
$$

The anti-deSitter $(\mathrm{AdS})$ cosmological constant $\Lambda=(3 / 4) \sigma^{2}$ arises from the last term in the scalar potential and the $\delta$-function brane tension $T=-2 \sigma$ effectively arises from 
the kinetic energy of $\phi$ and the first term of the supergravity scalar potential in (1). The tension and the cosmological constant are related as $\Lambda=(3 / 16) T^{2}$. This action is precisely the action of the type used in [3]; therefore the fine tuning between the AdS cosmological constant and the brane tension is, within our framework, a consequence of supersymmetry. (Recall that $\left.\sigma=(4 / 3) \lambda \eta^{3}\right)$.

For the fermionic sector, the action (11) becomes:

$$
\begin{aligned}
S_{\text {bulk }}^{\text {eff }} & =\int d^{4} e\left[-\frac{1}{2} R-i \bar{\chi} \bar{\sigma}^{m} \mathcal{D}_{m} \chi+\epsilon^{k l m n} \bar{\psi}_{k} \bar{\sigma}_{l} \tilde{\mathcal{D}}_{m} \psi_{n}\right. \\
& +\frac{1}{4}\left(i \epsilon^{k l m n} \psi_{k} \sigma_{l} \bar{\psi}_{m}+\psi_{m} \sigma^{n} \bar{\psi}^{m}\right) \chi \sigma_{n} \bar{\chi}-\frac{1}{8} \chi \chi \bar{\chi} \bar{\chi} \\
& +\frac{1}{2} \sigma \operatorname{sign} z\left(\psi_{a} \sigma^{a b} \psi_{b}+\bar{\psi}_{a} \bar{\sigma}^{a b} \bar{\psi}_{b}\right)-\frac{3}{4} \frac{\sigma}{\eta^{2}} \operatorname{sign} z(\chi \chi+\bar{\chi} \bar{\chi}) \\
& \left.+\frac{3}{4} \sigma^{2}\right] \\
S_{\text {brane }}^{\text {eff }} & =\int d^{4} e \delta(z)\left[-2 \sigma-\sqrt{2} \eta\left(\chi \sigma^{m} \bar{\sigma}^{z} \psi_{m}+\bar{\chi} \bar{\sigma}^{m} \sigma^{z} \bar{\psi}_{m}\right)\right. \\
& \left.+i \sqrt{2} \eta\left(\chi \sigma^{a} \bar{\psi}_{a}+\bar{\chi} \bar{\sigma}^{a} \psi_{a}\right)\right] .
\end{aligned}
$$

The limit $\eta \rightarrow 0$, implies that the fermionic fields $\chi, \psi_{m}$ do not contribute to the brane part of the effective action. In addition, in the bulk, the fermionic field $\chi$ has a large mass $(3 / 2)\left(\sigma / \eta^{2}\right) \rightarrow \infty$ and thus decouples from the theory. This loss of $\chi$ fermionic degrees of freedom parallels the fact that the kink, associated with the bosonic field partner, has been frozen to be "infinitely stiff" step function.

\section{Effective action from a supergravity model in D- dimensions}

In this section we employ an effective supergravity action in D-dimensions derived in [6] where most of the attention was focused on $\mathrm{D}=$ odd dimensions. The Lagrangian contains the graviton and gravitino as the only fields turned on in the supergravity multiplet, and the real scalar and a spin $1 / 2$ fermionic partner as the fields turned on in the matter supermultiplet; it can be viewed as a consistent truncation of a supergravity Lagrangian. (For the sake of simplicity we again focus on only one matter supermultiplet responsible for the formation of the wall.) Employing the supersymmetry transformations for the fermionic fields the authors of [6] obtained the equations of motion for the fermionic modes which can be lifted in a straightforward to obtain a supersymmetric Lagrangian, as described below.

The purpose of this section is to investigate the same issue as we did in the $\mathrm{D}=4$, $\mathrm{N}=1$ supergravity case by considering now a consistently truncated supergravity action 
in D-dimensions, with the supergravity multiplet $\left(e_{m}^{a}, \psi_{m}\right)$ and the scalar supermultiplet $(\phi, \chi)$. We focus on the thin wall limit of a thick smooth domain wall within this context.

Equations of motion for the Dirac fermion fields coupled to one real scalar field, a special case of the multi-field example studied in [6], are of the form:

$$
\begin{array}{r}
\Gamma^{m} \nabla_{m} \chi+M \chi+\frac{1}{2} \Gamma^{m} \Gamma^{n} \partial_{n} \phi \psi_{m}-W_{2} \Gamma^{m} \psi_{m}=0, \\
\Gamma^{m n p} \nabla_{n} \psi_{p}+W_{2} \Gamma^{m} \chi-(D-2) W \Gamma^{m n} \psi_{n}+\frac{1}{2}\left(g^{m n}-\Gamma^{m n}\right) \partial_{n} \phi \chi=0,
\end{array}
$$

where

$$
M=2(D-2) \frac{\partial^{2} W}{\partial \phi^{2}}-(D-2) W .
$$

These equations were shown to be invariant under the following supersymmetry transformations [6]:

$$
\begin{aligned}
\delta \psi_{m} & =\nabla_{m} \epsilon+W \Gamma_{m} \epsilon \\
\delta \chi & =\left(-\frac{1}{2} \Gamma^{m} \partial_{m} \phi+W_{2}\right) \epsilon
\end{aligned}
$$

where

$$
\nabla_{m} \epsilon=\partial_{m} \epsilon+\frac{1}{4} \omega_{m}^{a b} \Gamma_{a b} \epsilon, \quad \Gamma_{a b}=\frac{1}{2}\left[\Gamma_{a}, \Gamma_{b}\right] .
$$

In Ref. [6] the authors use the supersymmetry transformations (22)-(23) in order to construct a Nester tensor [9] and imposing the Witten condition $\Gamma^{m} \delta \psi_{m}=0$ [10] to ensure the stability of BPS backgrounds as it is usual in supergravity theories (and within the supergravity domain wall context first studied in [2]). The preservation of supersymmetry implies:

$$
\begin{aligned}
V(\phi) & =4(D-2)^{2}\left[\left(\frac{\partial W}{\partial \phi}\right)^{2}-\left(\frac{D-1}{D-2}\right) W^{2}\right], \\
W_{2} & =(D-2) \frac{\partial W}{\partial \phi} .
\end{aligned}
$$

As the next step we write down the consistently truncated supergravity action in Ddimensions (compatible with the above fermionic supersymmetry transformations (22)(23)). Employing results $1(19)-(20)$, (25)-(26) and also including both the "EinsteinHilbert" term and the kinetic part of the scalar field, yields:

$$
\begin{aligned}
S & =\int d^{D} x e\left[-R-g^{m n} \partial_{m} \phi \partial_{n} \phi-V(\phi)-\bar{\psi}_{m} \Gamma^{m n p} \nabla_{n} \psi_{p}-\bar{\chi} \Gamma^{m} \nabla_{m} \chi-M \bar{\chi} \chi\right. \\
& -\frac{1}{2} \partial_{n} \phi\left(\bar{\psi}_{m} \Gamma^{n} \Gamma^{m} \chi+\bar{\chi} \Gamma^{m} \Gamma^{n} \psi_{m}\right)-W_{2}\left(\bar{\psi}_{m} \Gamma^{m} \chi-\bar{\chi} \Gamma^{m} \psi_{m}\right) \\
& \left.+(D-2) W \bar{\psi}_{m} \Gamma^{m n} \psi_{n}+\text { four fermi terms }\right] .
\end{aligned}
$$

\footnotetext{
${ }^{1}$ Note that one has used the fact $\Gamma^{n} \Gamma^{m}=-\Gamma^{m n}+g^{m n}$ in the equation (20). This follows from the definitions $\left\{\Gamma^{m}, \Gamma^{n}\right\}=2 g^{m n}$ and $\Gamma^{m n}=\frac{1}{2}\left[\Gamma^{m}, \Gamma^{n}\right]$.
} 
We use the following notation: $\Gamma^{m n p} \equiv \Gamma^{[m} \Gamma^{n} \Gamma^{p]}, \Gamma^{m}=e_{a}^{m} \Gamma^{a}$, where $\Gamma^{a}$ are $2^{[D / 2]} \times$ $2^{[D / 2]}$ Dirac matrices and $e=\operatorname{det} e_{k}^{a}=\left|\operatorname{det} g_{m n}\right|^{1 / 2}$, with a mostly plus signature $(-++\ldots+)$.

\subsection{BPS domain wall and its thin limit}

The BPS domain wall background is obtained by solving Killing spinor equations, that is, by making (22)-(23) to vanish (Note the subsequent analysis is completely parallel to that of the previous section.):

$$
\begin{aligned}
\partial_{m} \epsilon+\frac{1}{4} \omega_{m}^{a b} \Gamma_{a b} \epsilon+W \Gamma_{m} \epsilon & =0, \\
\left(-\frac{1}{2} \Gamma^{m} \partial_{m} \phi+W_{2}\right) \epsilon & =0 .
\end{aligned}
$$

The bosonic Ansatz is of the form: the scalar field $\phi=\phi(z)$ and the metric:

$$
d s^{2}=e^{2 A(z)} \eta_{\mu \nu} d x^{\mu} d x^{\nu}+d z^{2}
$$

where $\mu, \nu=0,1,2, \ldots, D-2$ are world-volume (flat) indices and $z$ is the direction transverse to the wall.

With the above bosonic Ansatz eqs. (28) and (29) yield respectively:

$$
\partial_{z} A=\mp 2 W, \quad \partial_{z} \phi= \pm 2(D-2) \frac{\partial W}{\partial \phi} .
$$

The Killing spinors satisfy $\epsilon=e^{\frac{1}{2} A(z)} \epsilon_{ \pm}$, where $\Gamma^{z} \epsilon_{ \pm}= \pm \epsilon_{ \pm}$and $\Gamma_{\underline{z}} \epsilon_{ \pm}= \pm \epsilon_{ \pm}$. (The underlined indices refers to the tangent frame.)

With the $Z_{2}$ symmetric choice of the superpotential:

$$
W=\lambda\left(\frac{\phi^{3}}{3}-\eta^{2} \phi\right),
$$

i.e. its critical points transform into each other under $Z_{2}$ symmetry (for several examples of other superpotentials see, for instance, the references in [11]). Again, our focus will be on the thin wall limit $\lambda \rightarrow \infty, \eta \rightarrow 0$ and $\lambda \eta^{3} \rightarrow$ fixed.

The Bogomol'nyi equations (31) for the scalar field $\phi$ can be now written as:

$$
\partial_{z} \phi= \pm 2 \lambda(D-2)\left(\phi^{2}-\eta^{2}\right)
$$

which yields the domain wall solutions

$$
\phi_{ \pm}(z)=\mp \eta \tanh z / \Delta
$$

where $\Delta=1 / \lambda^{\prime} \eta$ specifies the domain wall width and $\lambda^{\prime}=2 \lambda(D-2)$. 
In the thin wall limit we have: $\lambda \rightarrow \infty, \eta \rightarrow 0, \lambda \eta^{3} \rightarrow$ fixed and $\Delta \rightarrow 0$. Now the solution (34) takes the form:

$$
\phi_{ \pm}(z)=\mp \eta \operatorname{sign} z
$$

In addition, the superpotential in this background solution is given by:

$$
W_{+}=-W_{-}=\left(\frac{2}{3} \lambda \eta^{3}\right) \operatorname{sign} z
$$

i.e. it is a step function with a finite width. Here $W_{ \pm}$stand for the first and second set of Bogomol'nyi equations (31). Regardless of the set of equations we choose we find the very same differential equation for the metric:

$$
\partial_{z} A=-\sigma \operatorname{sign} z, \quad \sigma=(4 / 3) \lambda \eta^{3} .
$$

As we shall see $\sigma$ is proportional to the wall (brane) tension.

The Bogomol'nyi equation (37) has the exact solution $A=-\sigma|z|$. Substituting this solution into the metric (30) we find

$$
d s^{2}=e^{-2 \sigma|z|} \eta_{\mu \nu} d x^{\mu} d x^{\nu}+d z^{2} .
$$

This is the metric used in the Randall-Sundrum scenario (in $D=5$ ) which is recognized as a $Z_{2}$ symmetric AdS background and allows for trapping of gravity on the domain wall.

Let us now compute the effective action for the bosonic background. The bosonic part of the action (27) can be written as :

$$
S=\int d^{D} x e\left\{-R-\left(\partial_{z} \phi\right)^{2}-4(D-2)^{2}\left[\left(\frac{\partial W}{\partial \phi}\right)^{2}-\left(\frac{D-1}{D-2}\right) W^{2}\right]\right\} .
$$

In order to derive the thin wall limit of this action, we employ the solution (34) and derive the following "identities":

$$
\begin{aligned}
& \partial_{z} \phi_{ \pm} \simeq \mp 2 \eta \delta(z), \quad\left[\partial_{z} \phi_{ \pm}(z)\right]^{2} \simeq \frac{4}{3} \lambda^{\prime} \eta^{3} \delta(z)=\sigma^{\prime} \delta(z), \quad \frac{\partial W\left(\phi_{ \pm}\right)}{\partial \phi} \simeq-\frac{\eta}{(D-2)} \delta(z), \\
& {\left[\frac{\partial W\left(\phi_{ \pm}\right)}{\partial \phi}\right]^{2} \simeq \frac{\sigma^{\prime}}{4(D-2)^{2}} \delta(z), \quad\left[W\left(\phi_{ \pm}\right)\right]^{2} \simeq \frac{4 \lambda^{2} \eta^{6}}{9}, \frac{\partial^{2} W\left(\phi_{ \pm}\right)}{\partial \phi^{2}} \simeq \mp 2 \lambda \eta \operatorname{sign} z .}
\end{aligned}
$$

We introduced new parameters $\lambda^{\prime}=2 \lambda(D-2)$ and $\sigma^{\prime}=2 \sigma(D-2)$. (Again, the expressions with delta functions should be understood to make sense only under integration.) In this case the action (39) takes the form of the following effective action:

$$
S^{e f f}=\int_{b u l k} d^{D} x e(-R+\Lambda)+T \int_{\text {brane }} d^{D} x e \delta(z),
$$


where

$$
\begin{aligned}
\Lambda & =(D-2)(D-1) \sigma^{2}, \\
T & =-4(D-2) \sigma,
\end{aligned}
$$

are the cosmological constant and the brane tension, respectively. They are related by

$$
\Lambda=\left(\frac{D-1}{D-2}\right) \frac{1}{16} T^{2}
$$

It is instructive to consider the case $D=5$. In this case we find $\Lambda=12 \sigma^{2}$, which is exactly the Randall-Sundrum fine-tuning relation. (Recall that in [3] one has $\Lambda \rightarrow$ $-\Lambda / 2, \sigma \rightarrow k$ and Planck mass $M_{p l} \neq 1$.)

Finally, the complete effective bulk and brane action can be written as:

$$
\begin{aligned}
S_{b u l k}^{e f f} & =\int d^{D} x e\left\{-R+\Lambda-\bar{\psi}_{m} \Gamma^{m n p} \nabla_{n} \psi_{p}-\bar{\chi} \Gamma^{m} \nabla_{m} \chi\right. \\
& \left. \pm g\left(\frac{6}{\eta^{2}}+1\right) \bar{\chi} \chi \pm g \bar{\psi}_{m} \Gamma^{m n} \psi_{n}+\text { four fermi terms }\right\}
\end{aligned}
$$

where $g$ is the gravitino mass given by $\mathrm{f}$ :

$$
g=\frac{\Lambda}{2(D-1) \sigma} \operatorname{sign} z
$$

and:

$$
\begin{aligned}
S_{\text {brane }}^{\text {eff }} & =\int d^{D} x e \delta(z)\left[T \pm \eta\left(\bar{\psi}_{m} \Gamma^{z} \Gamma^{m} \chi+\bar{\chi} \Gamma^{m} \Gamma^{z} \psi_{m}\right)\right. \\
& \left.+\eta\left(\bar{\psi}_{m} \Gamma^{m} \chi-\bar{\chi} \Gamma^{m} \psi_{m}\right)\right]
\end{aligned}
$$

We also see that in the thin wall limit the supersymmetry transformations (22) and (23) become

$$
\begin{aligned}
\delta \psi_{m} & =\nabla_{m} \epsilon \pm \frac{g}{(D-2) \sigma} \Gamma_{m} \epsilon \\
\delta \chi & =\left( \pm \Gamma^{z} \epsilon-\epsilon\right) \eta \delta(z) .
\end{aligned}
$$

From eq.(44) we notice that as $\eta \rightarrow 0$ (the thin wall limit) the mass of the fermion $\chi$ diverges because the term $6 / \eta^{2}$ goes to infinity. However, we can see clearly that in this limit the fermionic coupling in the brane action goes to zero as well. Thus, except for the four-fermi terms (whose detailed structure is not determined at the linearized level of the supersymmetry transformations), there is no other coupling between the fermions fields in the theory. Unlike the the graviton field, the fermion $\chi$

\footnotetext{
${ }^{2}$ Note that the gravitino mass mantain its sign by simply "rotating" the chiral fermion field with respect to the wall as $\psi_{ \pm} \rightarrow \pm \Gamma^{z} \psi_{ \pm}$.
} 
decouples from the theory due to its infinite mass. Furthermore as we can see in (48) the supersymmetry variation $\delta \chi$ does not have a contribution from the bulk, and the contribution from the brane has a prefactor that goes to zero.

As a special case of the effective theory in $D=5$, the term in the Lagrangian, corresponding to the gravitino mass is given by

$$
\mathcal{L}_{\psi^{2}}^{e f f}= \pm e\left(\frac{\Lambda}{8 \sigma} \operatorname{sign} z\right) \bar{\psi}_{m} \Gamma^{m n} \psi_{n}
$$

and the supersymmetry transformation (47) takes the form:

$$
\delta \psi_{m}=\nabla_{m} \epsilon \pm \frac{\Lambda \operatorname{sign} z}{24 \sigma} \Gamma_{m} \epsilon .
$$

Similar equations have been considered in the reference [8] in attempting to supersymmetrize the Randall-Sundrum action by including the term with "sign $z$ " by hand. Here, this term appear as a natural consequence of the thin wall limit of the thick wall BPS solution.

\section{Conclusions}

In this paper we focused on the structure of the supersymmetric effective action that is a thin wall limit of the thick BPS domain wall solution of $\mathrm{D}=4 \mathrm{~N}=1$ supergravity theory. We also explore a generalization to the D-dimensional, consistently truncated supergravities. In this limit, the kink solution for the scalar field $\phi$ provides a background for the delta-function stress-energy source for the thin wall and the

bulk corresponds to the AdS spacetime. In this limit the fermionic superpartner $\chi$ becomes infinitely heavy in the bulk and does not have a coupling to the graviton on the brane; it thus decouples from the theory. Furthermore, in the thin wall limit there is no fermionic contribution to the brane action. We have also explored the structure of the supersymmetry transformations in this limit, and arrived at the similar supersymmetry transformations [12] as in [8] (see also 13]), employed in attempts to supersymmetrize the Randall-Sundrum scenario; in our case these transformations are a natural consequence of taking a thin wall limit for a smooth BPS domain wall solution.

\section{Acknowledgments}

We would like to thank Neil Lambert, Eduardo Lima, Asad Naqvi, Christopher Pope, Gary Shiu and Kellog Stelle for helpful discussions. The work is supported in part 
by DOE grant DE-FG02-95ER40893 and NATO grant 976951. MC would like to thank SISSA, Italy for hospitality during the initial stages of the work. FAB would like to thank Department of Physics and Astronomy, University of Pennsylvania, for hospitality and Conselho Nacional de Desenvolvimento Científico e Tecnológico, CNPq, Brazil, for support.

\section{References}

[1] M. Cvetič and H.H. Soleng, Supergravity domain walls, hep-th/9604090, Phys. Rept. 282 (1997), 159.

[2] M. Cvetič, S. Griffies and S.-J. Rey, Static Domain Walls in $N=1$ Supergravity, hep-th/9201007, Nucl. Phys. B381 (1992), 301.

[3] L. Randall and R. Sundrum, An Alternative to Compactification, hepth/9906064, Phys. Rev. Lett. 83 (1999), 4690.

[4] K. Behrndt and M. Cvetič, Supersymmetric Domain-Wall World from D=5 Simple Gauged Supergravity, hep-th/9909058, Phys. Lett. B475 (2000), 253.

[5] K. Skenderis and P. K. Townsend, Gravitational Stability and Renormalization Group Flow, hep-th/9909070, Phys. Lett. B468 (1999), 46.

[6] G.W. Gibbons and N.D. Lambert, Domain Walls and Solitons in Odd Dimensions, hep-th/0003197, Phys. Lett. B488 (2000), 90.

[7] J. Wess and J. Bagger, Supersymmetry and Supergravity, Second Edition (Princeton University Press, 1992).

[8] A. Falkowski, Z. Lalak and S. Pokorski, Supersymmetrizing Branes with Bulk in Five-Dimensional Supergravity, hep-th/0004093, Phys. Lett. B491 (2000), 172.

[9] J.M. Nester, A New Gravitational Energy Expression with a Simple Positivity Proof, Phys. Lett. A83 (1981), 241.

[10] E. Witten, A Simple Proof of the Positive Energy Theorem. Comm. Math. Phys. 80 (1981), 381.

[11] J.D. Edelstein, M.L. Trobo, F.A. Brito and D. Bazeia, Kinks Inside Supersymmetric Domain Ribbons, hep-th/9707016, Phys. Rev. D 57 (1998), 7561; D. 
Bazeia and F.A. Brito, Bags, junctions, and networks of BPS and non-BPS defects, hep-th/9912015, Phys. Rev. D 61 (2000), 105019; D. Bazeia, J. Menezes and M. M. Santos, Complete Factorization of Equations of Motion in Supersymmetric Field Theories, hep-th/0103041.

[12] E. Bergshoeff, R. Kallosh and A. Van Proeyen, Supersymmetry in singular spaces, hep-th/0007044, JHEP 0010 (2000), 033.

[13] R. Altendorfer, J. Bagger and D. Nemeschansky, Supersymmetric RandallSundrum Scenario, hep-th/0003117. 